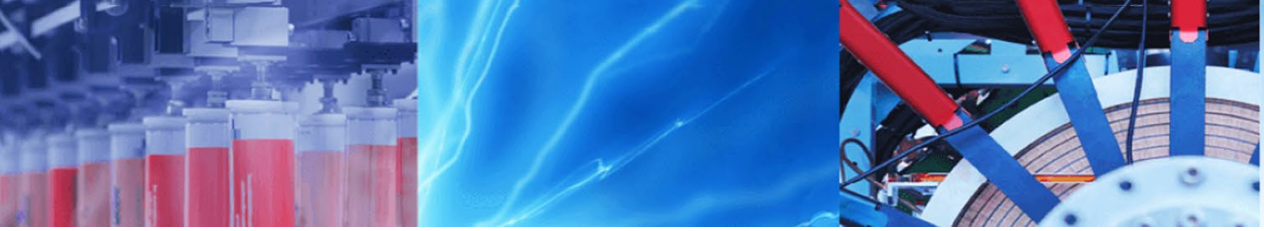

Research Article

\title{
Bioinspired designs for shock absorption, based upon nacre and Bouligand structures
}

\author{
George Raphel ${ }^{1}$ [ $\cdot$ Manu Moses Jacob ${ }^{1} \cdot$ Sreerag Viswanathan ${ }^{1}$
}

(c) Springer Nature Switzerland AG 2019

\begin{abstract}
Biological materials such as nacre, found in mollusk shells and Bouligand structures, found in the armor of many arthropods, exhibit values of toughness that are much higher than their constituent materials. This is achieved via specific arrangements and combinations of natural materials. In all biomimetic studies seen in literature so far, any one type of material alone is chosen for mimicry. The present work aims to create a set of biomimetic designs that incorporate macroscopic features of both nacre and Bouligand structures, so as to achieve toughness amplification in the given material. Finite element analysis (FEA) of relevant geometric designs are initially performed using ABAQUS software and experimental validation of the most promising model (which was found to show a $46.15 \%$ improvement over the reference model) is done. The testing method selected for both simulations as well as experiment is the Charpy impact test (ASTM E23).
\end{abstract}

Keywords Nacre $\cdot$ Bouligand structures $\cdot$ FEA $\cdot$ Biomimetics $\cdot$ Impact loading

\section{Introduction}

Nature, over the course of millions of years of evolution, is able to offer optimized solutions to many contemporary engineering problems. The field of biomimicry tries to draw upon these and adapt these solutions, as far as possible, to be used in synthetic systems. The present study is concerned with two prominent materials, namely nacre and Bouligand structures, which are found in biological structures whose functions are to provide structural integrity and protection from impact loads. The ability of these materials to show much higher values of toughness than those of their constituents has piqued the interest of the research community, as unlocking the mechanics behind the behavior of these materials can lead to the creation of structures with high values of specific strength and/or toughness.

\subsection{Nacre}

Nacre, found in the shells of mollusks, in spite of being composed of $95 \%$ volume of brittle ceramic, boasts a toughness that is much higher than its constituent material [1]. It consists of a brick and mortar arrangement of Aragonite plates (bricks) in a protein matrix (mortar), as shown in Fig. 1. The protein layer can be further classified into two types, namely the 'interlaminar' and 'intralaminar' layers. The former is found between adjacent layers of bricks, while the latter is found in the gap between adjacent tablets [2]. Nacre is found right below the hard outer calcite layer of the mollusk shell, as a fine coating. As in most biological growth processes, the tablets are formed by mineralization of Aragonite at specific nucleation sites, and as such, each layer of bricks exhibits Voronoi tiling [3].

During an impact event (usually normal to the plane of the nacre layers), the individual tablets tend to slide away from each other (Fig. 2). The displacement of the tablets

George Raphel, georgeraphel@me.ajce.in | 'Department of Mechanical Engineering, Amal Jyothi College of Engineering, Kanjirappally, Kerala 686518, India.

SN Applied Sciences (2019) 1:1022 | https://doi.org/10.1007/s42452-019-1062-7

Received: 6 May 2019 / Accepted: 8 August 2019 / Published online: 12 August 2019 


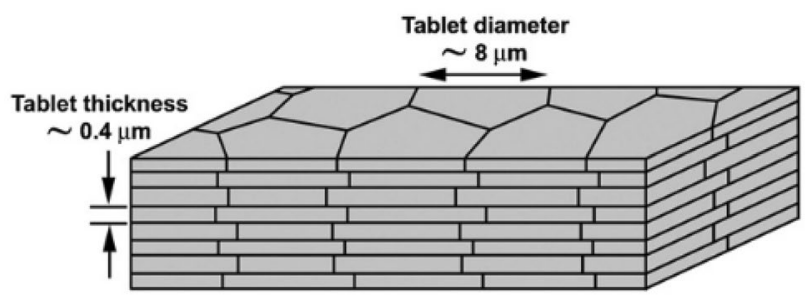

Fig. 1 A schematic diagram of the microstructure of nacre, showing the brick and mortar arrangement of ceramic tiles in a protein matrix. The thickness of the protein layer between adjacent tablets is $50 \mathrm{~nm}[1]$

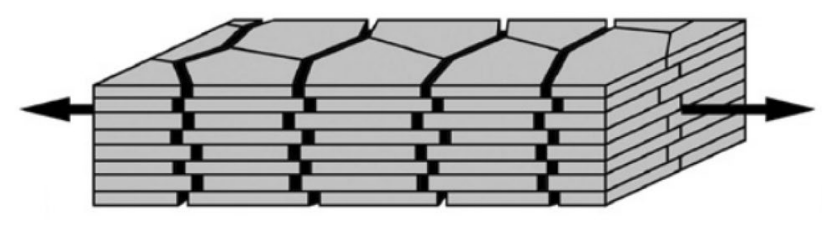

Fig. 2 Schematic showing tablet sliding. The tablet separation generates voids at the end of the tablets [1]

leads to the dissipation of the impact energy, thereby resulting in toughness amplification.

Barthelat et al. showed that each layer of nacre has a long range order surface waviness, which led to the formation of a dovetail joint with tablets from adjacent layers. This feature was primarily responsible for the strain hardening and toughness amplification in nacre, during tablet sliding [1]. Along with the surface waviness, nano-asperities on the tablet surfaces and mineral bridges between overlapping tablets also contribute to strain hardening and a corresponding increase in toughness. Zhang et al., with the help of molecular dynamics simulations, showed that the behavior of the protein layer enabled the growth of intra granular cracks in nacre, which increases the toughness of nacre, through crack extension [4]. At the nanoscale, Wang et al. studied the effect of intra crystalline organics, present as inclusions in nacre, and showed that these modify the crack propagation paths, thereby increasing toughness [5].

\subsection{Biomimetic structures of nacre}

Many researchers have attempted to manufacture nacremimetic materials. But so far, none of the designs are feasible for large scale manufacturing. The most suitable method for capturing the geometry of nacre is 3D printing. But the limitations in usable materials, and the difficulty to print metals is a cause for concern. Askarinejad et al. [6], Zhang et al. [7] and Gu et al. [8, 9] used 3D printing to create samples for experimental studies, while Mirkhalaf et al.
[10] used a doctor blading technique to fabricate nacre mimetic specimen. Other methods include freeze casting, infiltration and lithography techniques [11]. But each of these methods have their respective shortcomings, which prevents them from being used for manufacturing nacre mimetic materials on a larger scale. Kakisawa et al. through a powder processing method using glass fibers, coated with silver as the raw material, created nacre mimetic composites that could be used in structural applications [12], while Bouville et al. proposed a method for the production of nacre mimetic bulk ceramics [13].

\subsection{Bouligand structures}

Another example of a toughness amplification is observed in the dactyl limbs of the Mantis shrimp, as shown in Fig. 3. The structure consists of different zones, comprised of different kinds of arrangements of crystalline and amorphous hydroxyapatite. The forces generated upon impact from the club are high enough to break aquarium glass ( $500 \mathrm{~N})$ [14]. Despite multiple impacts, there is no catastrophic failure of the limb, which indicates a very high specific toughness. The major energy absorbing component is region II (shown in Fig. 3c), which consists of helicoidally arranged fibrous composite layers, called Bouligand structures, first identified by Bouligand [15]. A SEM image of its cross section is shown in Fig. 3d, while a 3D model of the material is shown in Fig. 3e. Upon impact, cracks propagate along a helicoidal path through the material, thereby leading to considerable energy dissipation, through multiple mechanisms [16]. A theoretical model was created for crack propagation by Suksangpanya et al. [16].

Ribbans et al. showed that fiber-matrix modulus ratios and pitch angles of helical reinforcements greatly influenced the interlaminar shear strength of helicoidal fibril structures [17].

\subsection{Biomimetic structures of dactyl limbs}

Grunenfelder et al. [18] showed that helicoidal carbon fiber-epoxy composites reduced through thickness damage propagation in laminates during impact, thereby improving the toughness of the composite. Mirkhalaf et al. conducted analytical, numerical and experimental studies on 3D printed helicoidal specimen subjected to impact loads, in which the crack propagation paths were controlled [19]. The biomimetic models were found to have improved fracture toughness over conventional laminate specimen. Ginzburg et al. [20] conducted numerical studies of helicoidal composites subject to Low Velocity Impact $(\mathrm{LVI})$, and found that helicoidal composites had the least amount of fiber damage, post-impact. 


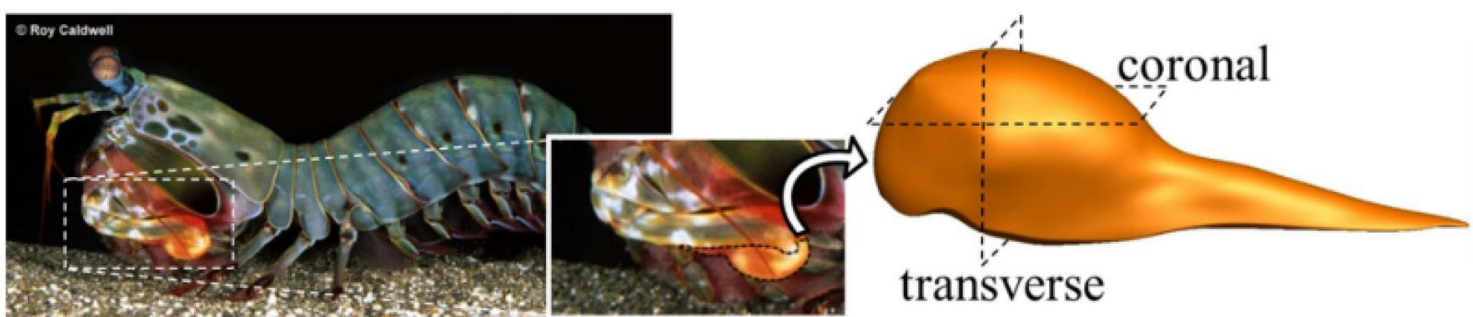

(a)

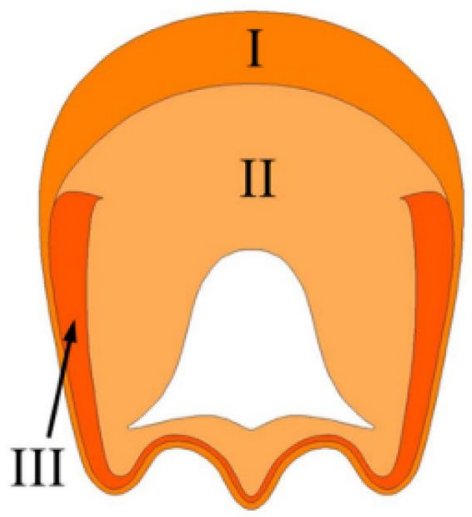

(c)

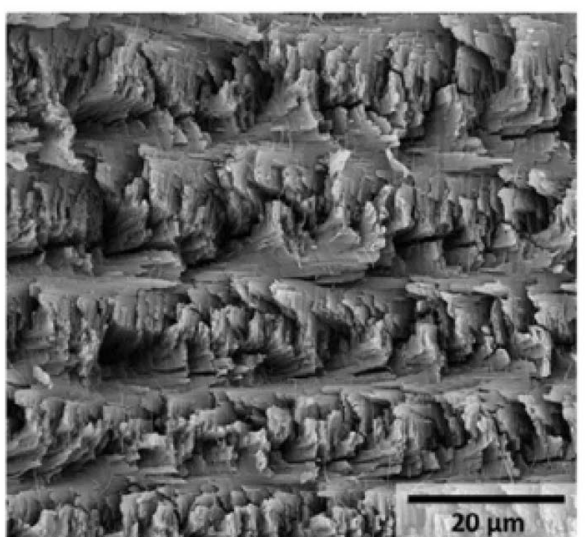

(d) (b)

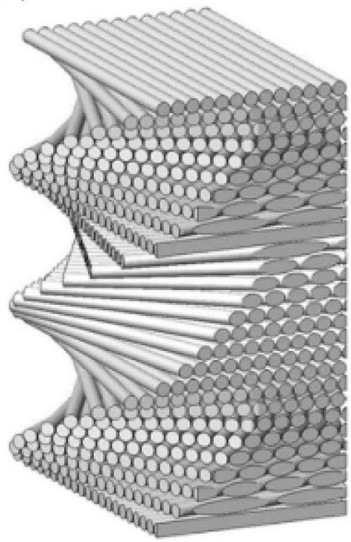

(c)

Fig. 3 a The Mantis shrimp, showing its dactyl club (inset), b 3D model of the club, $\mathbf{c}$ transverse section of the club, $\mathbf{d}$ SEM image of a fractured surface, e 3D illustration of Bouligand structures [16]

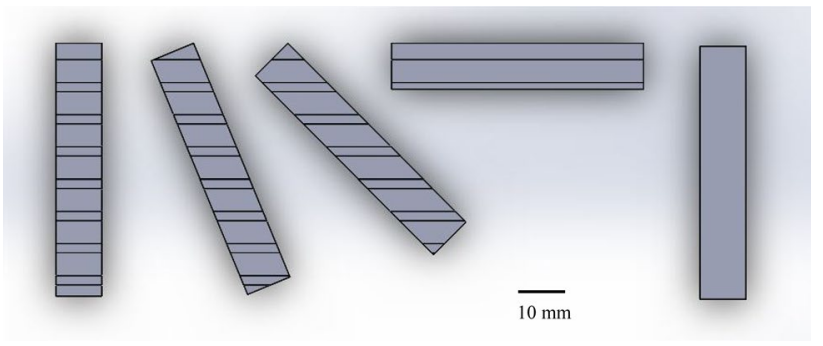

Fig. 4 The geometry of each layer of a sample synthetic, bioinspired metallic specimen. In this sample, the width of each groove is $2 \mathrm{~mm}$, while the depth is $1 \mathrm{~mm}$

\subsection{Scope of the current study}

The present study is an attempt to combine the salient features of nacre and Bouligand structures, so as to produce a specimen with superior toughness compared to its constituent materials. As of the time of submission of this article, no such work had been found in published literature, wherein a combination of multiple natural structures, with similar functions was studied.

In this work, each horizontal layer of the geometry of nacre is reproduced at the millimeter scale by machining grooves onto $2 \mathrm{~mm}$ thick metallic plate, as shown in Fig. 4. The reason for the selection of a $2 \mathrm{~mm}$ thick plate as the base material is that a thinner plate has a tendency to warp during machining, while a thicker plate would make handling the material more difficult, while at the same time, decrease the number of layers. In case a nonconventional method such as laser cutting is employed, the plate thickness may be reduced, and the number of layers increased, which should (theoretically) give better results. But the improvements cannot be justified by the corresponding rise of manufacturing costs (especially when it comes to manufacture of large specimens for use in practical applications).

All outer dimensions of the cut pieces correspond to the requirements of an ASTM E23 Charpy impact specimen (55 $\mathrm{mm} \times 12.7 \mathrm{~mm}$ ).

The grooves act as joints between the bulk portions of the plate (similar to the protein layer between adjacent ceramic tablets in nacre). The aspect ratio of natural nacre is $20: 1$, but that of the thicker metallic portions of the synthetic model are kept at 5:1 in order to facilitate ease of manufacture, as a higher aspect ratio at these length scales would cause premature bending and/or oversized tablets.

The orientations of adjacent layers of the geometry were varied by equally spaced angular distances, in such a manner as to obtain an angular difference of 90 degrees between the first and last layers. This is similar 
to the arrangement of composite layers in natural Bouligand structures. Although the angle of twist lies within 10 degrees for natural structures, the synthetic layers are inclined at much larger angles ( 22.5 degrees), due to the lower number of layers in the synthetic model.

In this work, fifteen different geometries are created and analyzed using Finite Element Analysis (FEA). All specimens of interest were either directly taken from available designs in literature, or are a modification of previously studied designs. The selection criterion was the ease of manufacture using conventional manufacturing processes.

A material model validation study is performed using the most promising geometry, the results of which are given in the next section. This is followed by a comparative study of different kinds of proposed geometric combinations.

\section{Material model validation}

The specimen selected for validation is subjected to a Charpy impact test (ASTM E23) [21]. An initial Finite Element Analysis of different geometries are performed using ABAQUS Explicit (ver. 6.13), so as to identify the optimum arrangement, and the optimized geometry is validated experimentally. The details of the FEA study are given in the following sections. The material chosen was Aluminum due to its ease of availability in different sizes in the market and machinability (which in turn, drastically brings down the cost of production of the required geometries), whose properties were taken from literature [22], and modelled based upon the Johnson-Cook ductile damage model [23].

The geometry of the model, subject to experimental validation is shown in Fig. 5 .

Each layer consists of a $2 \mathrm{~mm}$ thick aluminum sheet, with $1 \mathrm{~mm}$ deep grooves, machined on it. Although nacre tablets are composed of brittle ceramic, aluminum was chosen as the material due to its ease of availability and good machinability. Also, the dimensions of the grooves were selected in such a manner so as to keep them within the minimum resolution of equipment available in the industry; the goal is to be able to manufacture these specimen using commonly available industrial tools, without the need for specialized/custom equipment.

The orientations of the grooves are (bottom to top) are $0,+45,90,-45,0$ degrees. The grooves create weak joints between stiffer tablet (full thickness) sections, thereby mimicking the arrangement of nacre. The helicoidal twisting of the grooves mimics the arrangement of laminae in Bouligand structures.

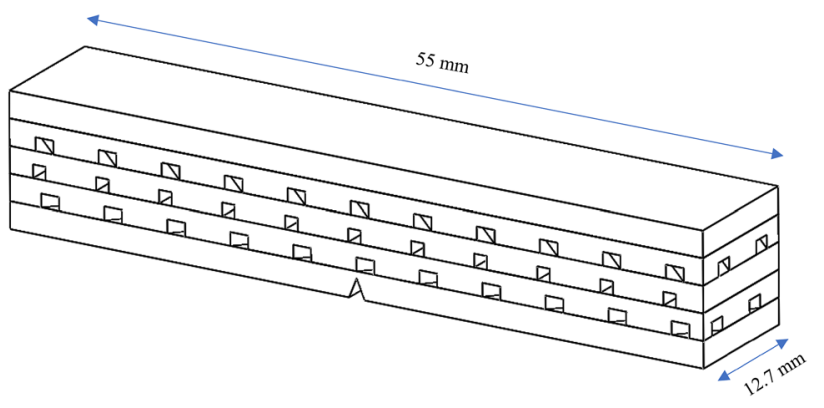

Fig. 5 The geometry for material data validation. Each layer of metal is $2 \mathrm{~mm}$ thick, while the overall dimensions are selected as per the requirements of the ASTM E23 standard. The grooves in the second (from top), third and fourth layers are oriented at $-45,90$ and +45 degrees respectively

\subsection{Finite element analysis}

Finite Element Analysis was performed using the commercial package, SIMULIA ABAQUS Explicit (version 6.13), a product of Dassault Systèmes. A mesh of size $0.25 \mathrm{~mm}$ consisting of C3D8R elements was used. These 3-dimensional reduced integration elements are suitable for explicit analyses due to better stability of each iteration. Although convergence was obtained at a mesh size of $\sim 1.75 \mathrm{~mm}$ itself, an additional reduction in size was made, in order to improve the accuracy of the results, considering the high amount of geometric and contact non-linearities in the model. The average time taken for a $2 \mathrm{~mm}$ mesh model was about $4 \mathrm{~h}$, while that for that for the $0.25 \mathrm{~mm}$ mesh model was about $10 \mathrm{~h}$. The impactor was modeled as a rigid body, using R3D3 and R3D4 elements.

The meshed model is shown in Fig. 6. The impactor and the supports are modelled as shell bodies, with reference points, denoted by 'RP'. The laminate specimen is placed as a stack in the supports, and the impactor is given a linear velocity, into the plane of the top surface. The supports are given an 'Encastre' boundary condition ( 0 degrees of freedom), while the hammer tip is constrained to move linearly, along the line of impact (normal to the top face of the material).

A grid independent study was performed on the model (Fig. 7) using mesh sizes varying from 0.5 to $2.75 \mathrm{~mm}$, with the maximum von Mises stress in the specimen as the variable (shown in Fig. 6). It may be observed that convergence is obtained at a mesh size of $1.75 \mathrm{~mm}$ itself. But, a smaller mesh size $(0.25 \mathrm{~mm})$ has been used in all further models, so as to account for accurate geometric modelling of fine features, as well as nonlinear effects at the contact regions.

A frictional contact (with a frictional coefficient of 0.3 ; arbitrarily chosen at first, and subsequently validated by the experimental results) was provided between the 


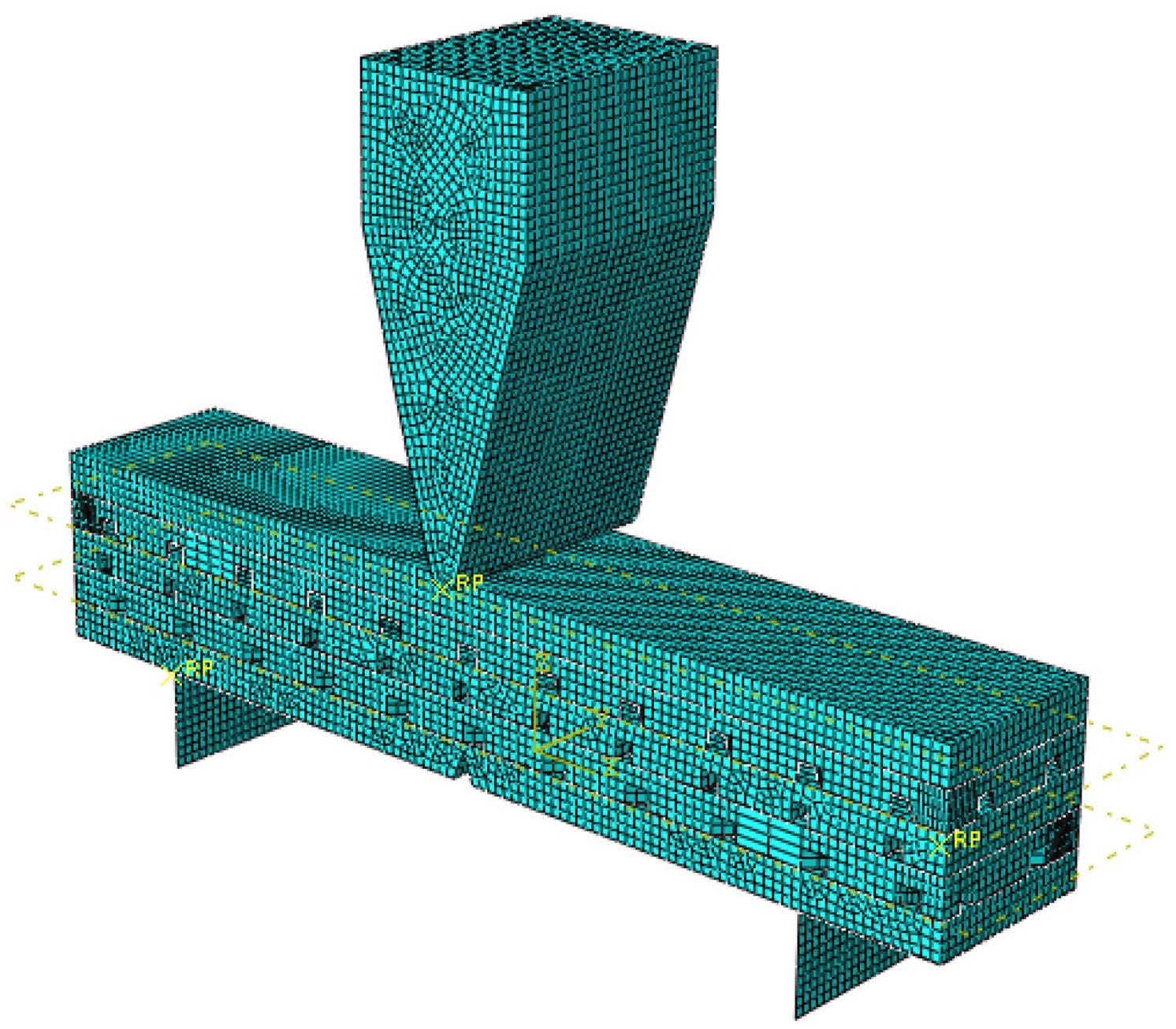

Fig. 6 The mesh of the assembled components. The specimen is placed between the hammer tip (top) and the supports (bottom plates)

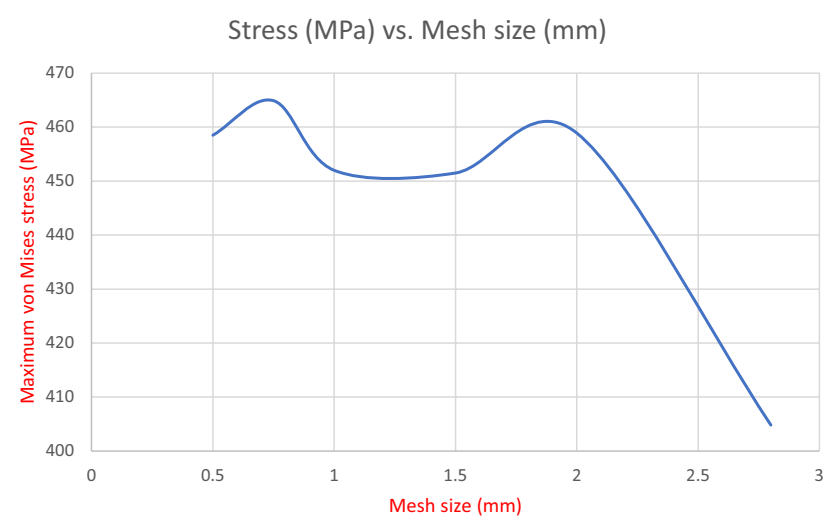

Fig. 7 Mesh convergence graph, showing the variation of maximum von Mises stress as a function of average element size. Convergence is initially obtained at a mesh size of $1.75 \mathrm{~mm}$ itself different layers using the 'General Contact' formulation in ABAQUS/Explicit, and an initial velocity of $5.92 \mathrm{~m} / \mathrm{s}$ (which was the velocity of the hammer tip, when it struck the specimen in the experimental model) was assigned to the impactor head, to simulate the drop from a height of $1 \mathrm{~m}$. The mass of the hammer was $17.12 \mathrm{~kg}$, and the step time, $0.005 \mathrm{~s}$ (which is the time required for complete penetration of the hammer tip into the material). The results of the FEA study is shown in Fig. 8. The von Mises stress is considered here due to the complex nature of the geometry, and the loading conditions. Since it is not easy to predict in advance the type of deformation in the material during/ after impact, it is better to consider the von Mises stress over the principal stresses.

\subsection{Experimental validation}

The optimized specimen was validated by fabrication of the specimen, followed by testing in an impact testing 

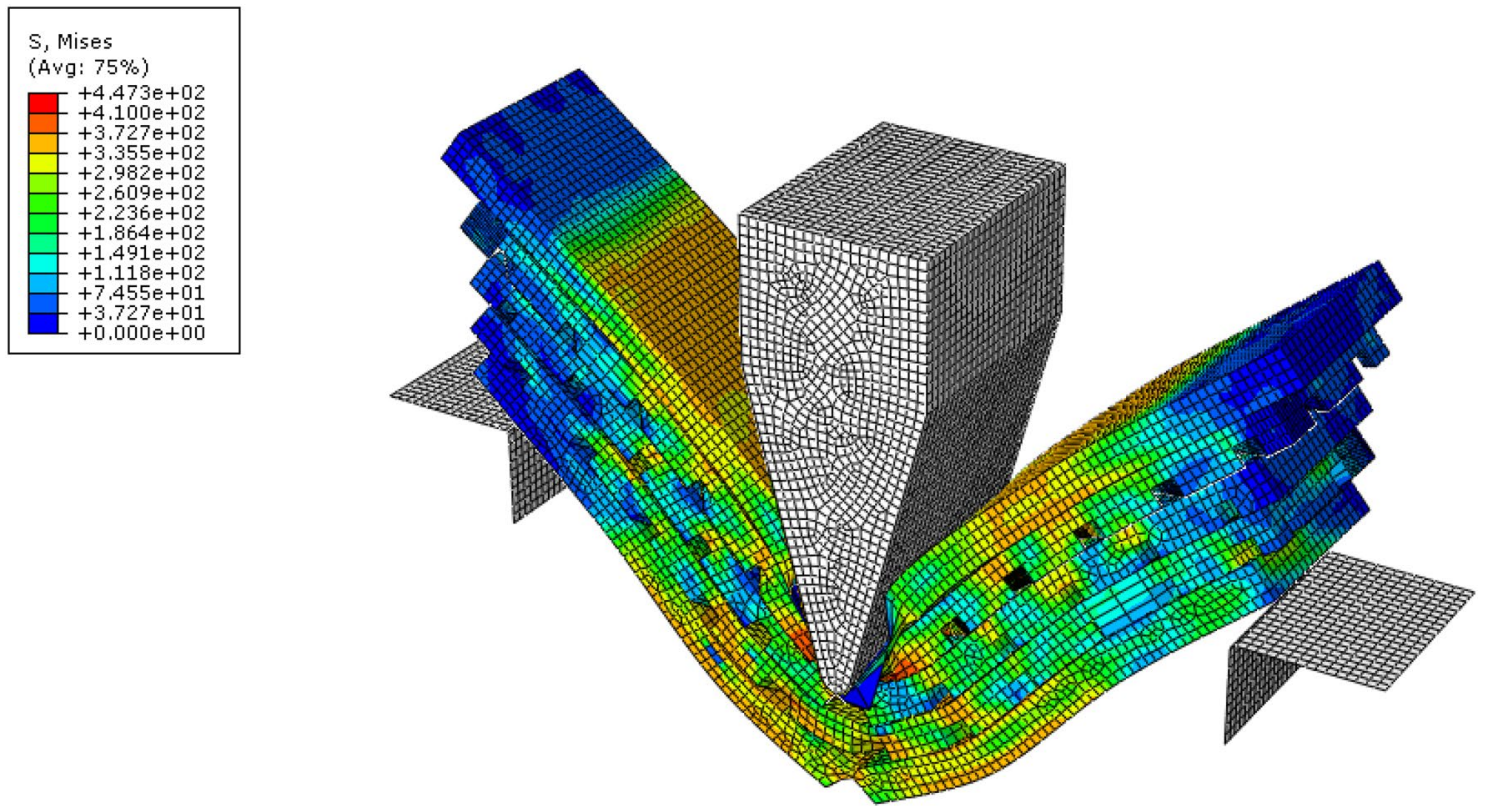

Fig. 8 von Mises stress distribution at the end of the step time. It may be noted that the Mises stress has been distributed throughout each layer of material, instead of being concentrated at the

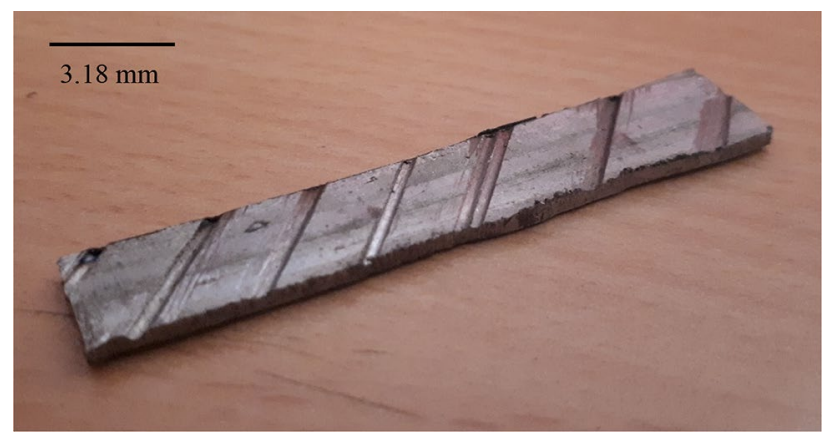

Fig. 9 A single plate unit ( $45^{\circ}$ slotted). The thickness and depth of each slot is $2 \mathrm{~mm}$, while the overall dimensions of the plate correspond to ASTM E23 standards

machine, manufactured by FIE (Fuel Instruments \& Engineers Pvt. Ltd.), with a maximum capacity of $300 \mathrm{~J}$. The specimen was fabricated by machining aluminum sheets of thickness $2 \mathrm{~mm}$ (Fig. 9), and then laying them out in stacks. In order to keep the plates in contact, the entire arrangement was wrapped up in adhesive tape. The fabricated specimen, prior to and after impact testing are shown in Figs. 10 and 11, 12, respectively. The energy absorbed by tape rupture is negligible ( $5 \%$ of the impact energy), when compared to the energy absorbed by the specimen, and can be safely neglected. A certain amount of randomness was brought in via slightly staggered arrangement of the plates, and varying thickness at the edges of the laminates. center portions alone (as seen in a bulk material, subject to similar loading conditions)

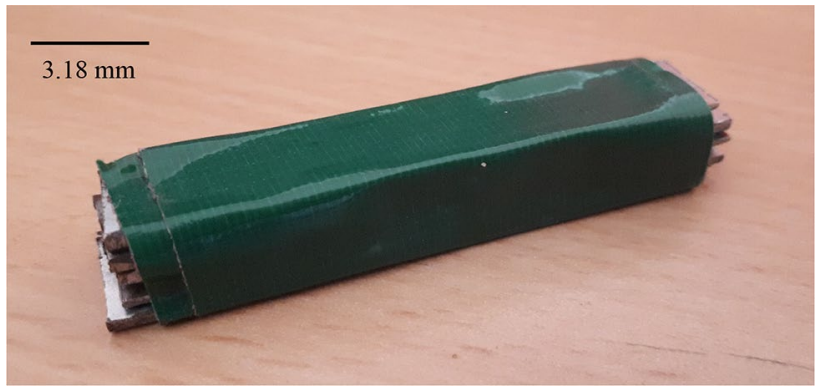

Fig. 10 The fabricated specimen, prior to testing. The layers of metal have been enclosed in a layer of adhesive tape, in order to maintain the shape of the specimen, during the testing process, as only frictional contact is considered amongst the layers

Upon impact, the energy absorbed by the specimen was found to be $38 \mathrm{~J}$. This is a $46.15 \%$ increase over the base value of bulk aluminum alloy, which is $26 \mathrm{~J}$ [24]. The energy absorbed by the FEA model was found to be $36.60 \mathrm{~J}$. The difference between experimental and FEA values is $3.68 \%$, which is within the acceptable limit (generally $10-30 \%$, depending upon the software and type of analysis).

A comparison of the shapes of the deformed specimens (Figs. 11, 12) show that there is reasonable agreement between FEA and experimental values. At the same time, spring back of the specimen (observed in the experimental specimen) is neglected in the FEA study, in order to simplify the process. Also, unlike the experimental model, the

\section{SN Applied Sciences}


Fig. 11 The figure given here shows the FEA simulation results of the experimentally validated specimen. It may be noted that unlike a standard Charpy test in which the test specimen gets split into two separate pieces, in the case of the laminate model, the specimen is highly deformed, yet remains as a single unit

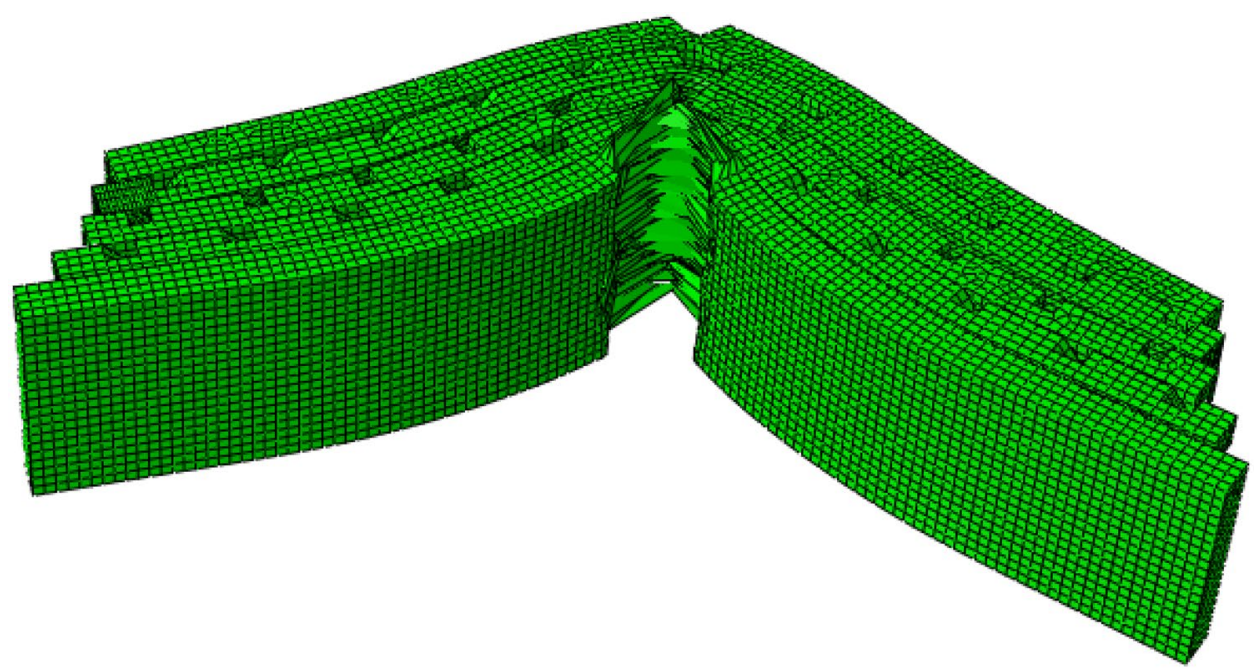

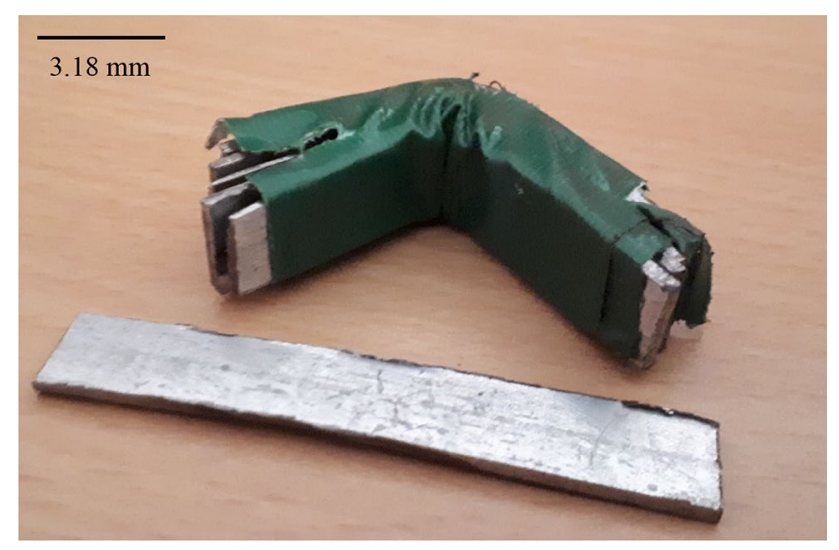

Fig. 12 The deformed specimen (experimental result) is shown here. Unlike the FEA result, some amount of springback of the laminates are observed in the experimental specimen. A single, undeformed laminate layer has been placed before it, in the photograph, in order to have a rough idea about the extent of deformation in the specimen

FEA model does not have any toss energy associated with it (Toss energy is the energy used up when the specimen gets thrown off the support, during experimental testing. This phenomenon does not occur in the FEA analysis, as accounting for this energy loss would require considerably larger analysis times 10 times more and is not worth the computational effort). Moreover, the impactor head, being a rigid body, does not absorb any energy at all.

\section{Biomimetic study of combination geometries}

Different types of geometries were designed (shown in Table 1) and studied using FEA simulation of a Charpy impact test.
All specimens were subjected to in plane normal impact by a rigid body impactor, having a point mass of $2.10 \mathrm{~kg}$, travelling at an initial velocity of $7 \mathrm{~m} / \mathrm{s}$, which are the values used in experimental tests of high-grade ballistic armor steel [25]. The results of the bioinspired geometries were compared with a reference solid block of the same material, subject to similar loading conditions.

The final geometries that were obtained by relevant combinations can be grouped into two categories:

\subsection{Bulk material geometries (B1-B9)}

Initially, a set of models were created where the only features were the presence of cylindrical holes in a bulk Charpy specimen. The objective of studying these blocks were to understand the effects of removing material from different regions of the bulk material and check if such a procedure could yield a desirable level of toughness amplification.

These focus upon the manufacturability aspect of the specimen. The sizes of the specimen are maintained at the order of a few millimeters and the components are designed in way that allows for manufacturing on a very large scale, using existing, conventional techniques.

It consists of different specimens, each drilled with varied arrangements of $5 \mathrm{~mm}$ diameter (minimum size of cutting tool available in most CNC machines in industry) holes/elongated holes. The position of the holes, the depth of cut and orientation are varied, so as to create multiple models.

It may be noted that the bulk specimens are intended to be used for the purpose of comparison with the laminate specimens (explained in the subsequent sections), and no in depth analyses have been performed upon these samples. For such a study would be too generic and is not within the scope of the present research. 
Table 1 Summary of finite element analysis of different specimens, and the energy absorbed by each

\begin{tabular}{ll}
\hline Model ID Image & $\begin{array}{l}\text { Energy } \\
\text { absorbed } \\
(\mathrm{J})\end{array}$ \\
\end{tabular}

Reference (R)

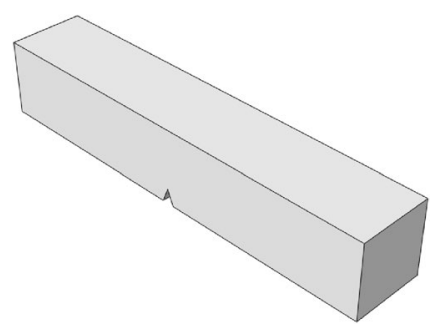

Bulk model (B1)

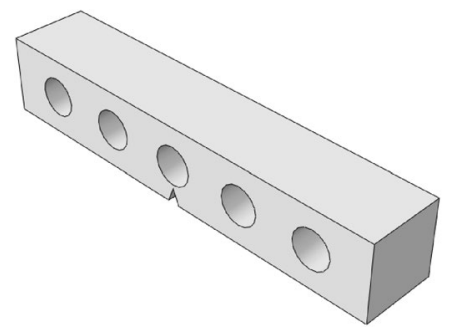

B2

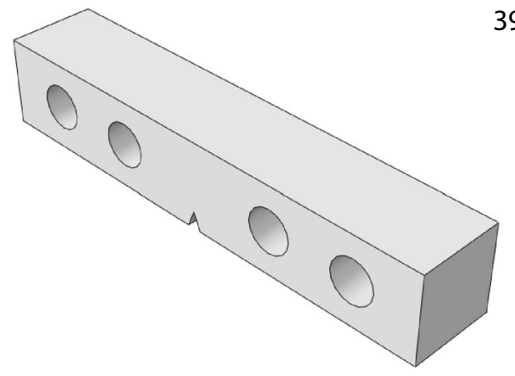

B3

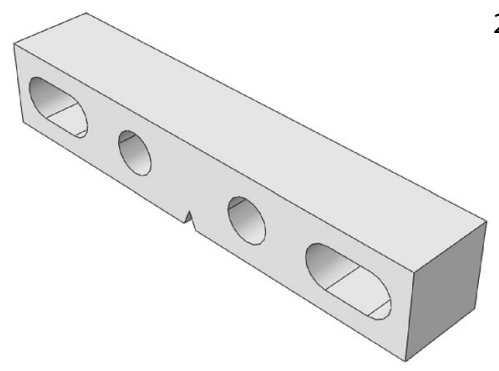

B4

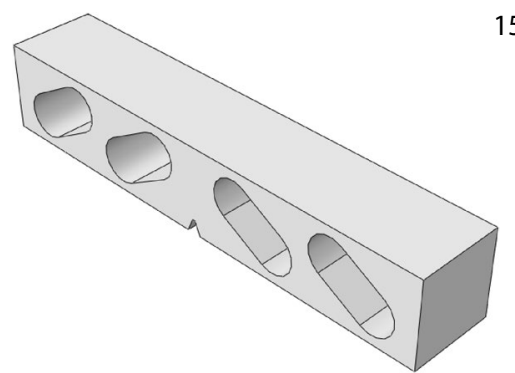

Table 1 (continued)

Model ID Image

Energy

absorbed

(J)

B5

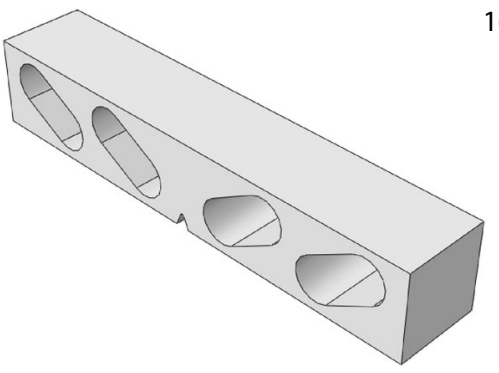

B6

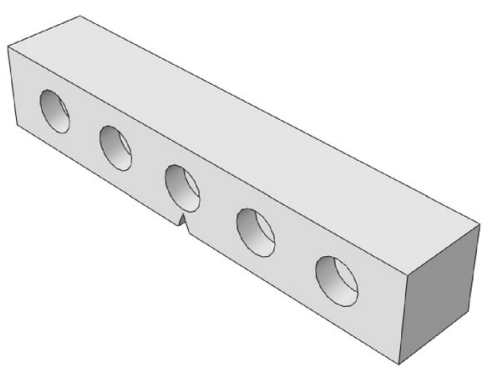

B7

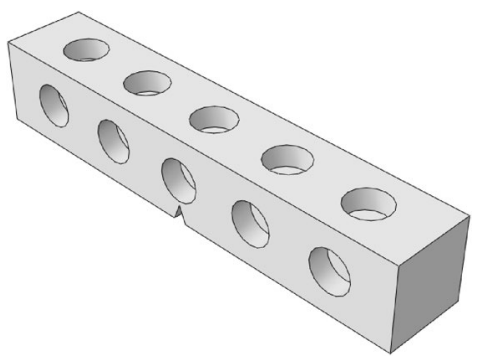

B8

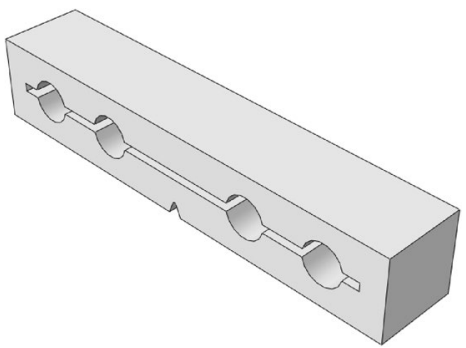

B9

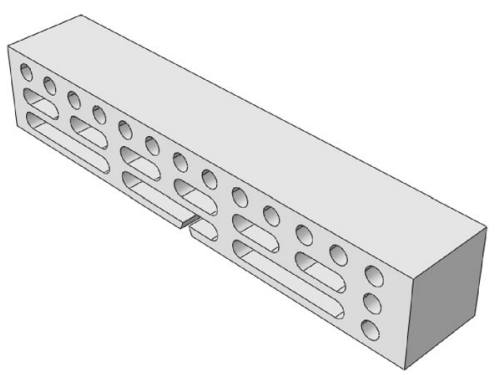

19.44
24.87

14.70

8.48 
Table 1 (continued)

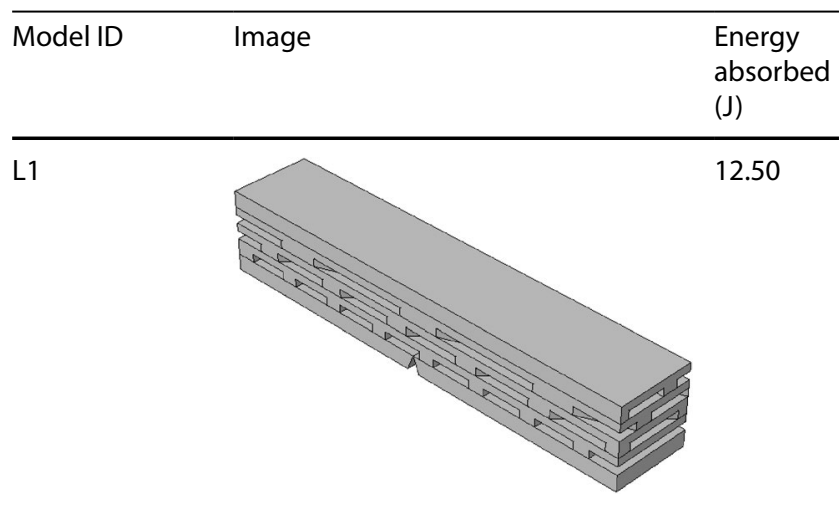

$\mathrm{L} 2$

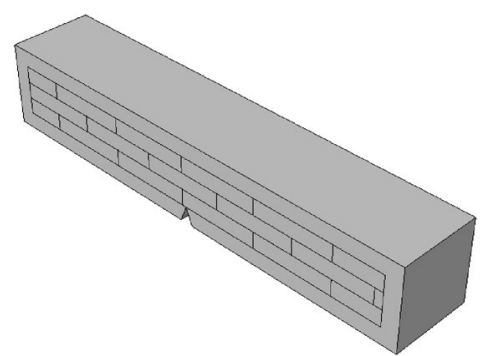

12.63

L3

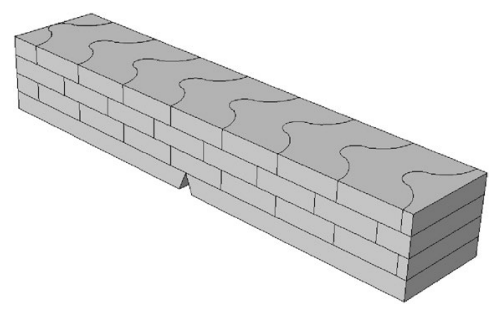

L4

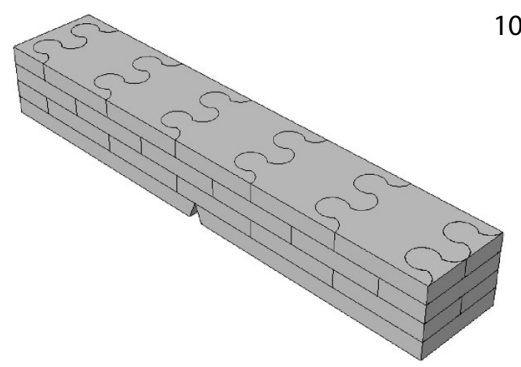

L5

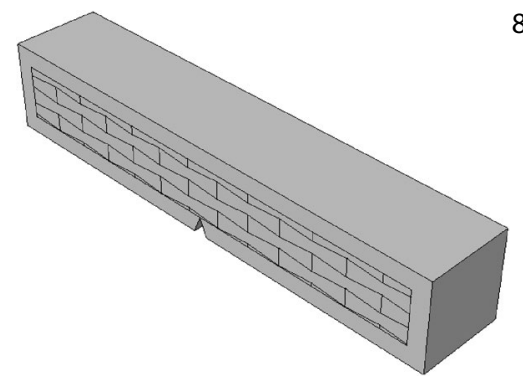

8.75

The bulk specimens are prefixed by ' $B$ ', while the laminate specimens are prefixed by ' $L$ '

\subsection{Laminate geometries (L1-L5)}

Another method is to create the same geometry using stacks of sheet metal, in order to form a laminated specimen. The advantage here is that smaller features can be modelled as grooves or features on the sheet metal.

This is the proposed method to create truly nature mimetic geometries.

All laminate geometries (L1-L5) were designed using existing works in literature that mimic nacre, as a starting point [26-30]. All nacre mimetic models stipulate a brick and mortar combination of ceramic tablets, surrounded by a viscoelastic polymer layer. Due to the difficulties of modelling, as well as manufacture of dual material models, only single material models have been considered in this study. This study intends to check for the presence of toughness amplification at these dimensions (millimeter scale). So far, no study has been performed on bioinspired specimens at these scales. The results of this work pertain to a feasibility study of such an approach, and may be used to form the basis for further parametric studies at these scales. Upon verification of this, dual material models may also be considered in subsequent works. The protein interface between adjacent nacre tablets have been replaced by weak joints (grooves in the laminate, which create weak links). The protein layer between adjacent tablet layers has been eliminated completely, and frictional resistance alone is present here.

Some of these models were subsequently modified to incorporate related features, from other, suitable geometries such as Bouligand structures [14, 20,31-34] and biological sutures [25]. Bouligand structures were mimicked by orienting each layer of nacre mimetic aluminum plates at a slightly different angle in the plane of the lamina, while sutures were created as jigsaw interlocks between adjacent tablets in the same plane $(\mathrm{L} 3, \mathrm{~L} 4)$.

Another set of geometries involves jigsaw-like interlocking between adjacent pieces of metal, in the same plane, while another involves projections on the surface of each plate, which lock into grooves at the bottom of the adjacent plate. These two types create a form of strain hardening mechanisms in the material. The corresponding features in nacre are surface waviness of the tablets (forming dovetail joints) and mineral bridges [1].

A set of models based upon work by Al-Maskari et al. has also been included for the analysis [26]. In this model, each lamina (which consists of a row of individual bricks) has a triangular wave shape at the boundaries. This causes tablet interlocking and resistance to relative sliding of adjacent layers (analogous to strain hardening in natural nacre). 


\section{Results and discussion}

Finite Element Analysis was conducted upon different geometric configurations (shown in Table 1), and the energy absorbed by each specimen when subject to a Charpy Impact test was noted.

The reference specimen shows a value of $26.30 \mathrm{~J}$, which is a $1.16 \%$ deviation from the experimental value [24], and is within acceptable error limits, thereby further validating the material model used.

In order to have a better comparison amongst the geometric models, a normalized chart of total energy absorption as well as specific energy absorption (energy absorbed per unit mass of material) has been plotted (Fig. 13). The values are normalized with respect to those of the reference model (R).

\subsection{Bulk models}

From the normalized chart, it may be observed that models B2 and B3 show properties that are better than the reference specimen, i.e., these are the models that show significant toughness amplification. Model B8 shows better specific properties than the reference model.

In the case of B2 and B3, it may be observed that maximum material is present in the region that lies along the striking path of the pendulum hammer. At the same time, material may be removed from the edges of the specimen, so as to improve its specific properties. At the center, the specimen behavior is similar to that of the bulk specimen. But the presence of voids on the side portions allows for some movement. At the same time, the irregular geometry allows for multiple reflection of stress waves within the specimens, similar to the phenomenon observed in the hyoid structure of a woodpecker's head [35]. This is not possible in the reference model, which provides a smooth, straight path for the propagation of any stress waves that are generated during the dynamic impact event.

Generally speaking, in all the bulk models, energy absorption occurs only in the regions that lie within a $20 \mathrm{~mm}$ radius, away from the point of initial impact and most of the remaining portion of the material does not contribute significantly to the toughness of the specimen.

Even in the case of the functionally graded model, the presence of a gradually varying set of features (holes) does little to contribute to toughness amplification. This may be attributed to the large dimensions of the features, when compared to natural systems (tree trunks, plant stems, nacre etc.).

\subsection{Laminate models}

All the laminate models show a decrease in energy absorption by about half (on average). Among the laminates, L2 shows high energy absorption, while L1 shows excellent specific characteristics, as L2 is a closely packed structure and $\mathrm{L} 1$ has voids in between.

But the best improvement was shown by the experimentally validated model. This may be attributed to the smaller size of the grooves in the model, when compared to L1.

On the other hand, in L1 (Fig. 14), the stress at the V notch tends to remain at a uniform value of $\sim 400 \mathrm{MPa}$, even though there is a drastic failure of the top portion (in contact with the hammer tip). This phenomenon, apart
Fig. 13 Normalized total and specific energy absorption of different geometries, from FEA studies. The specimens with a'B' prefix denote the bulk models, while those with ' $\mathrm{L}$ ' denote the laminate models. All energy values have been normalized with respect to that of the reference block

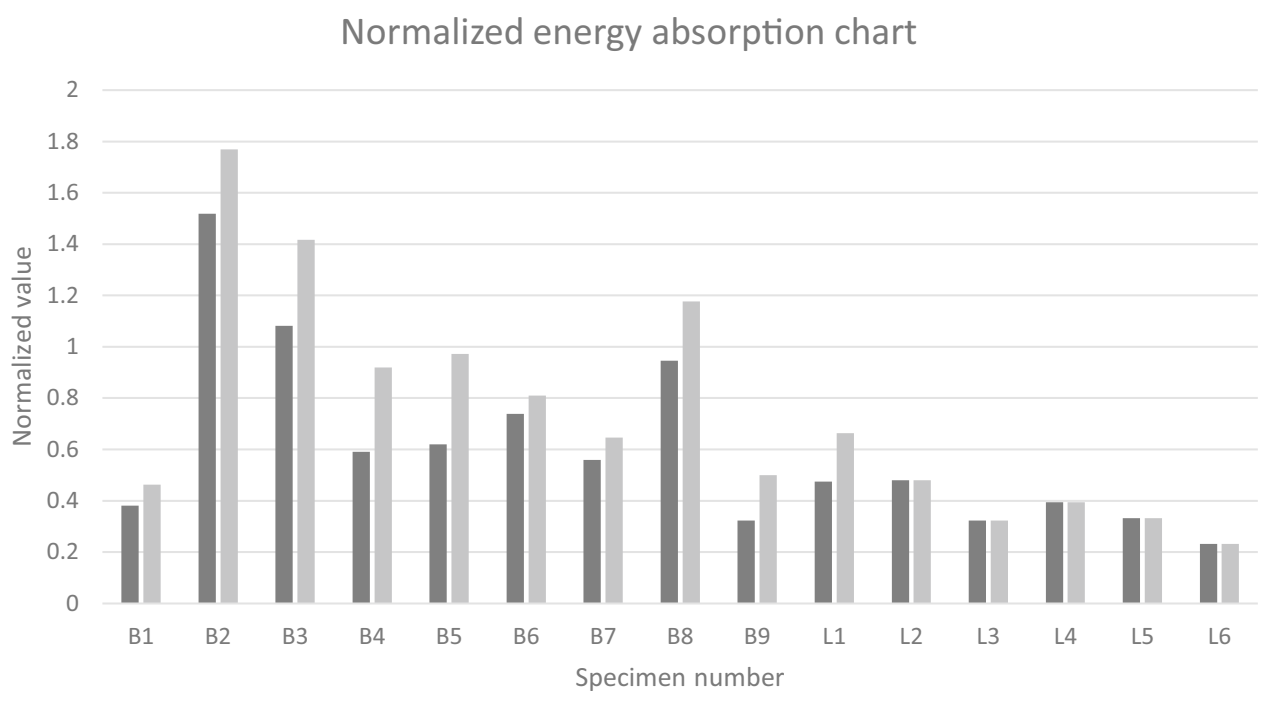

normalized total energy absorption Normalized specific energy absorption 
Fig. 14 Variation of Mises stresses at different pints of the L1 (laminate) specimen. The stress at the $V$ notch remains approximately constant $(\sim 400 \mathrm{MPa})$, while that at the top surface decreases with the progress of time. A change in stress is indicative of damage to the material, which is seen on the top surface, but does not progress to the bottom surface of the specimen

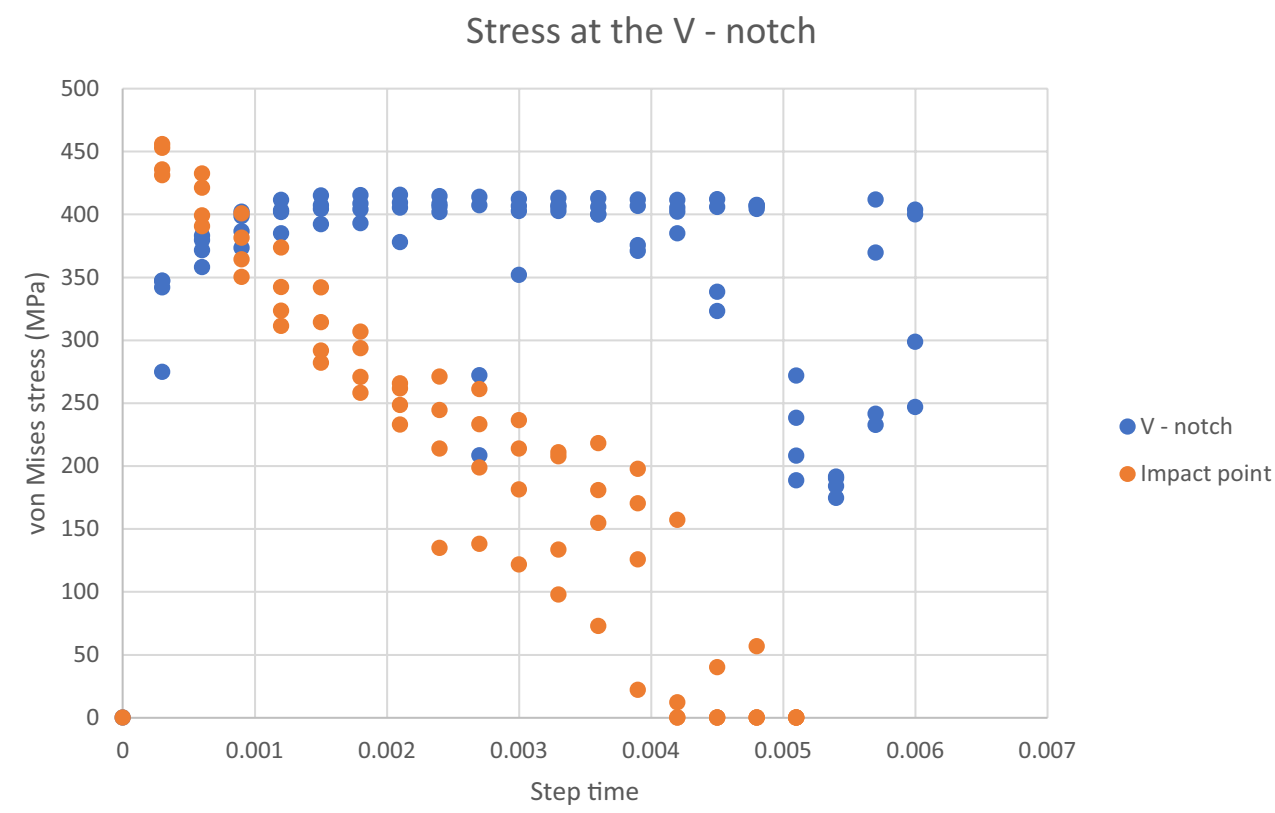

from indicating failure at the top surface, also shows that the bottom layer does not fail, but is still intact, i.e., damage in the material is arrested, prior to reaching the lower layers of the model, which is the case with natural armor systems. It may be noted that unlike the bulk specimen, no fracture occurs at the notch. This indicates that despite the presence of a major stress concentration point, the model is able to avoid catastrophic failure, as is the case with bulk specimens.

In the case of jigsaw structures ( $L 3, \mathrm{~L} 4)$, elastic deformations which occurred at the sutures appeared to lead to weakening and rapid disintegration of the entire structure. A decrease in the size of the sutures can lead to a solution to this problem, but it comes at the cost of manufacturability. Hence, no further studies were conducted in this aspect.

The Al-Maskari model (L5) also had a similar problem as the jigsaw models. An absence of the viscoelastic interface could be the reason for the lower energy absorption observed.

Also, while fracture is the dominant form of energy absorption in the bulk specimens, the biomimetic laminate specimens use elasto-plastic deformations as the dominant form of energy absorption. This characteristic is useful when designing structures that need to maintain structural integrity, in spite of being damaged by impact loads.

Thus, even though the energy absorbed for the improved laminate specimen (L1) is not significantly different from the reference specimen, the main advantage lies in the use of relatively lesser material, and effective redistribution of the stresses within the material. While the bulk specimen exhibits brittle failure at the midpoint (with failure initiating at the V-notch), a more ductile behavior is observed in the case of the laminate specimen, as seen from the FEA and experimental results. This is advantageous when creating light-weight materials that are required to act as protective barriers against impact forces.

\section{Conclusion}

In the present work, a set of novel geometries have been proposed, which try to implement a combined biomimicry of nacre and Bouligand structures. From the FEA and experimental results, it is observed that some of the new designs indeed shows improved toughness, when compared to the bulk material (46.15\% improvement in energy absorption, for the experimentally validated specimen).

Moreover, sheet metal manufacturing and machining systems can be set up very easily in industry and thus, the proposed design can be manufactured very easily and in an economic manner; no special tools or equipment are required for the same. This work is an initial step towards making biomimetic systems feasible for manufacturing using existing methods.

At the same time, parametric studies need to be conducted in future works to ascertain the optimum combination of dimensions, groove spacing, orientation and number of layers required for maximum toughness amplification. Another probable course of future work could be the inclusion of adhesives or viscoelastic material between the layers of metal plates, which could improve the toughness of the structure. Also, other types of tests, such as Low Velocity Impact (LVI) tests need to be performed to 
make further assessment of the delamination behaviour and failure mechanisms in these materials.

Prospective applications of this material include improved shock absorption systems, with higher specific strength, as well as bullet proof armour materials.

Acknowledgements This work has been supported by the Amal Jyothi Scholars' Community for Inspiring Innovation (ASCII), at Amal Jyothi College of Engineering, Kerala, India.

\section{Compliance with ethical standards}

Conflict of interest The authors have no conflict of interest as far as the present study is concerned, to the best of their knowledge.

\section{References}

1. Barthelat F, Tang H, Zavattieri PD, Li CM, Espinosa HD (2007) On the mechanics of mother-of-pearl: a key feature in the material hierarchical structure. J Mech Phys Solids 55:306-337

2. Stempflé $P$, Bourrat $X$, Rousseau M, Lopez E, Takadoum J (2013) Nanotribology of nacre: anisotropic dissipation in a multiscale hybrid material. Tribol Int 63:250-264

3. Rousseau M et al (2005) Sheet nacre growth mechanism: a Voronoi model. J Struct Biol 149(2):149-157

4. Zhang N, Yang S, Xiong L, Hong Y, Chen Y (2016) Nanoscale toughening mechanism of nacre tablet. J Mech Behav Biomed Mater 53:200-209

5. Wang S, Zhu X, Li Q, Wang R, Wang X (2016) Damage-tolerance strategies for nacre tablets. J Struct Biol 194:199-204

6. Askarinejad S, Choshali HA, Flavin C, Rahbar N (2018) Effects of tablet waviness on the mechanical response of architected multilayered materials: modeling and experiment. Compos Struct 195:118-125

7. Zhang P, Heyne MA, To AC (2015) Biomimetic staggered composites with highly enhanced energy dissipation: modeling, 3D printing, and testing. J Mech Phys Solids 83:285-300

8. Gu GX, Libonati F, Wettermark SD, Buehler MJ (2017) Printing nature: unraveling the role of nacre's mineral bridges. J Mech Behav Biomed Mater 76:135-144

9. Gu GX, Takaffoli M, Buehler MJ (2017) Hierarchically enhanced impact resistance of bioinspired composites. Adv Mater 29(28):1-7

10. Mirkhalaf $M$, Barthelat $F$ (2016) Nacre-like materials using a simple doctor blading technique: fabrication, testing and modeling. J Mech Behav Biomed Mater 56:23-33

11. Huang C, Cheng Q (2017) Learning from nacre: constructing polymer nanocomposites. Compos Sci Technol 150:141-166

12. Kakisawa $H$, Sumitomo $T$, Inoue $R$, Kagawa $Y$ (2010) Fabrication of nature-inspired bulk laminar composites by a powder processing. Compos Sci Technol 70(1):161-166

13. Bouville F, Maire E, Meille S, Van De Moortèle B, Stevenson AJ, Deville $S$ (2014) Strong, tough and stiff bioinspired ceramics from brittle constituents. Nat Mater 13(5):508-514

14. Wegst UGK, Bai H, Saiz E, Tomsia AP, Ritchie RO (2015) Bioinspired structural materials. Nat Mater 14(1):23-36

15. Bouligand $Y$ (1972) Twisted fibrous arrangements in biological materials and cholesteric mesophases. Tissue Cell 4(2):192-217

16. Suksangpanya N, Yaraghi NA, Kisailus D, Zavattieri P (2017) Twisting cracks in Bouligand structures. J Mech Behav Biomed Mater 76(June):38-57

SN Applied Sciences
17. Ribbans B, Li Y, Tan T (2016) A bioinspired study on the interlaminar shear resistance of helicoidal fiber structures. J Mech Behav Biomed Mater 56:57-67

18. Grunenfelder LK et al (2014) Bio-inspired impact-resistant composites. Acta Biomater 10(9):3997-4008

19. Mirkhalaf M, Tanguay J, Barthelat F (2016) Carving 3D architectures within glass: exploring new strategies to transform the mechanics and performance of materials. Extrem Mech Lett 7:104-113

20. Ginzburg D, Pinto F, lervolino O, Meo M (2017) Damage tolerance of bio-inspired helicoidal composites under low velocity impact. Compos Struct 161:187-203

21. D6110 (2015) Norma E23-07a-Standard test methods for notched bar impact testing of metallic materials. Am Soc Test Mater Handb 14(C):28

22. Flores-Johnson EA, Shen L, Guiamatsia I, Nguyen GD (2014) Numerical investigation of the impact behaviour of bioinspired nacre-like aluminium composite plates. Compos Sci Technol 96:13-22

23. Johnson GR, Cook WH (1985) Fracture characteristics of three metals subjected to various strains, strain rates, temperatures and pressures. Eng Fract Mech 21(1):31-48

24. Abubakre OK, Mamaki UP, Muriana RA (2015) Investigation of the quenching properties of selected media on 6061 aluminum alloy. J Miner Mater Charact Eng 08(04):303-315

25. Malik IA, Mirkhalaf M, Barthelat F (2017) Bio-inspired'jigsaw'-like interlocking sutures: modeling, optimization, 3D printing and testing. J Mech Phys Solids 102:224-238

26. Al-Maskari NS, McAdams DA, Reddy JN (2017) Modeling of a biological material nacre: waviness stiffness model. Mater Sci Eng C 70:772-776

27. Rim JE, Zavattieri P, Juster A, Espinosa HD (2011) Dimensional analysis and parametric studies for designing artificial nacre. J Mech Behav Biomed Mater 4(2):190-211

28. Abid N, Pro JW, Barthelat F (2018) Fracture mechanics of nacre-like materials using discrete-element models: effects of microstructure, interfaces and randomness. J Mech Phys Solids 124:350-365

29. Ni Y, Song Z, Jiang H, Yu SH, He L (2015) Optimization design of strong and tough nacreous nanocomposites through tuning characteristic lengths. J Mech Phys Solids 81:41-57

30. Gao C, LiY (2019) Mechanical model of bio-inspired composites with sutural tessellation. J Mech Phys Solids 122:190-204

31. Naleway SE, Porter MM, McKittrick J, Meyers MA (2015) Structural design elements in biological materials: application to bioinspiration. Adv Mater 27(37):5455-5476

32. Li L, Weaver JC, Ortiz C (2015) Hierarchical structural design for fracture resistance in the shell of the pteropod Clio pyramidata. Nat Commun 6:6216

33. Tadayon M, Amini S, Masic A, Miserez A (2015) The mantis shrimp saddle: a biological spring combining stiffness and flexibility. Adv Funct Mater 25(41):6437-6447

34. Cheng L, Thomas A, Glancey JL, Karlsson AM (2011) Mechanical behavior of bio-inspired laminated composites. Compos Part $A$ Appl Sci Manuf 42(2):211-220

35. Johnson KL et al (2014) Geometric effects on stress wave propagation. J Biomech Eng 136(2):021023

Publisher's Note Springer Nature remains neutral with regard to jurisdictional claims in published maps and institutional affiliations. 